\title{
Equivalence of Dual Graphs
}

\author{
M. Azram, Member, IACSIT
}

\begin{abstract}
Because of interesting and useful geometric as well as topological properties, alternating knots (links) were regarded to have an important role in knot theory and 3-manifold theory. Many knots with crossing number less than 10 are alternating. It was the properties of alternating knots that enable the earlier knot tabulators to construct tables with relatively few mistakes or omissions. Graphs of knots (links) have been repeatedly employed in knot theory. This article is devoted to establish relationship between knots and planar graphs. This relationship not only enables us see the equivalence of the graphs corresponding to black regions and the dual graph corresponding to white regions.
\end{abstract}

Index Terms-Dual graphs, LR graphs, planar isotopy, $\mathbf{R}^{*}$-move, reidmeister moves.

\section{INTRODUCTION}

Knots have been introduced as early as is the story of Alexander and Gordian but never got scientific status till Nineteen Century. Once the Kelvin's Theory of Vortex Atoms was discarded, the knot theory merges as a part of Physics and then relegated to Mathematics. Mathematicians were perplexed at the seemingly unending number of ways a knot could be shaped and turned. Consequently, these give rise to the central problem of knot theory i.e., whether two knots (links) are equivalent or not especially whether a knot is equivalent to its mirror image or not. This was the motivation for much of the recent work in knot theory, which is devoted to search for invariants of knots. Reidemeister moves, tricoloring, knot polynomials (Alexander polynomial, Jones polynomial, Bracket polynomial, Homfly polynomial, Kauffman polynomial, etc.) are few examples. The study of invariants underwent in a kind of phase transition, which has linked knot theory to chemistry, molecular chemistry, mathematical physics, particles physics, polymer physics, statistical mechanics, fluid mechanics, kinematics, $\mathrm{C}^{*}$-algebra, conformal field theory, crystallography, cryptography, graph theory, computer systems and networks, etc. In the recent past, biologists and chemists studying genetics discovered an exciting link of knot theory with DNA (genetic material of all cells, containing coded information about cellular molecules and processes) and synthetic chemistry [1], [2]. DNA is just one application of knot theory, which presently is an area of intense mathematical activities worldwide.

Because of interesting and useful geometric as well as topological properties, alternating knots (links) were regarded to have an important role in knot theory and

Manuscript received March 10, 2013; revised May 16, 2013.

M. Azram is with the Department of Science, Faculty of Engineering, IIUM, Kuala Lumpur 50728, Malaysia (e-mail: azram50@ hotmail.com). 3-manifold theory. Many knots with crossing number less than 10 are alternating. It was the properties of alternating knots that enable the earlier knot tabulators to construct tables with relatively few mistakes or omissions. It is conjectured that as the crossing number increases, the percentage of knots that are alternating goes to 0 exponentially quickly [3]. This article is devoted to establish relationship between knots and planar graphs. This relationship not only enables us see the equivalence of the graphs corresponding to black regions and the dual graph corresponding to white regions.

\section{MATERIAL AND METHOD}

Knots (links) will be confused with their projections. By the planar isotopy we mean the motion of the projection in the plane that preserves the graphical structure of the underlying universe. Two knots (links) in space can be deformed into each other (ambient isotopy) if and only if their projections can be transformed into one another by planar isotopy and the three Reidemeister moves. Two knots are equivalent (via Reidemeister moves) denoted by the symbol $\sim$, if and only if (any of) their projections differ by a finite sequence of Reidemeister moves [4]. Ambient isotopy and equivalence via Reidemeister moves is the same [5]. A connection between knot theory and graph theory has firstly been established by Reidemeister [4]. Graphs of knots (links) have been repeatedly employed in knot theory [6]-[8]. A knot (link) diagram can be considered as a planar graph with 4-valent vertices. We will call such a planar graph the universe of a knot (link). Kauffman [9] has established that "Universes of knots (links) are in one-to-one correspondence with planar graphs". Azram [10] has extended the same by establishing that the "Connected universes of knots (linked links) are in one-to-one correspondence with connected planar graphs".

For the construction of the graph, shade (checker-board shading) the regions of knot (link) as black and white. Associate a pseudo graph to the knot (link) so that the vertices of the graph correspond to the black regions and the edges of the graph correspond to the crossings shared by the black regions. We will call this graph as the graph corresponding to black regions of the knot (link). See Fig. 1.

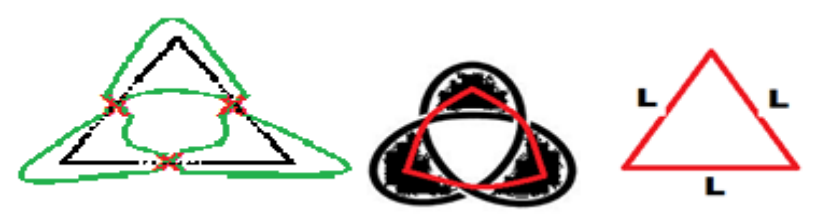

Fig. 1. Construction of the graph corresponding to black regions of knot (link). 
The construction of the graph corresponding to white regions is exactly the same, all one needs to consider white regions instead of black regions. The LR-Graph is pseudo graph corresponding to the black (white) regions, where the edges are labeled as "L" or " $R$ " depending on whether the upper string at the corresponding crossing falls on the left or on the right side when going from either black (white) region to the other adjacent black (white) region. If $\mathrm{G}$ be the graph corresponding to black (white) regions of a given knot (link) then by the "dual graph" of G, we mean the graph corresponding to the white (black) regions of the same knot (link). The unique choice of over/under structure for the crossings makes the knot as an alternating. LR-graph corresponding to a reduced alternating knot is always connected, planar, loop less, and bridgeless graph with all the labeling as " $L$ " or " $R$ ". The result that "Connected universes of knots (linked links) are in one-to-one correspondence with connected planar graphs" can be generalized as "knots (linked links) are in one-to-one correspondence with connected planar LR-Graphs" [11]. This construction of LR-Graphs and vice versa does not require signed graphs and so is the orientation.

\section{RESULTS AND DISCUSSION}

In the future, knots will be confused with their class of projections with crossings indicated unless otherwise stated. Terminology, definition and concepts for most of the material are standard. By the planar isotopy we mean the motion of the knot projection in the plane that preserves the graphical structure of the underlying universe. The pivotal moves in the theory of knots are the Reidemeister moves. We will view these moves as Reidemeister moves of type I, II, and III. Fig. 2.

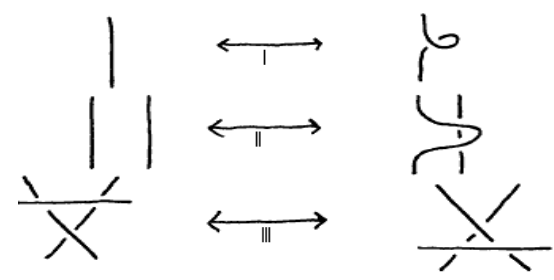

Fig. 2. Reidemeister moves of type I, II and III.
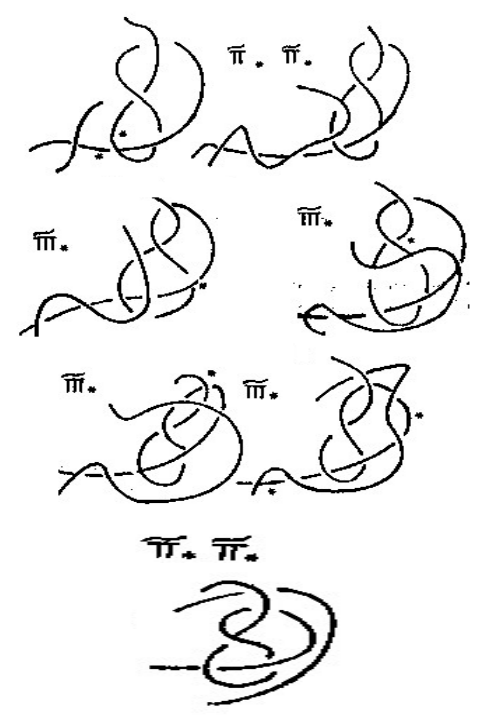

Fig. 3. Illustration of $\mathrm{R}^{*}$-move.
The results and discussion in this article are variational, diagrammatic and illustrative. The $\mathrm{R}^{*}$-move, which is a generalized form of Reidemeister move of type II, will be a pivotal move in the discussion hereafter. Before establishing $\mathrm{R}^{*}$-move, let us consider the following example, Fig. 3. Note that by $\underset{a^{*}}{\sim}$ we mean that a move of type ' $\bullet$ ' is performed at the location ' $*$ '.

Theorem 1. $\mathrm{R}^{*}$-move is well defined via Reidemeister moves, that is,

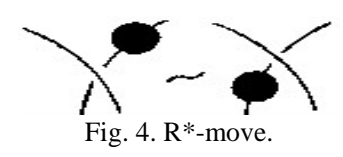

Proof. Without loss of generality, assume the part $\bullet$ of the knot (link) has n number of crossings. Let $i_{1}$ be the very first crossing encountered while going from left to right and $i_{n}$ be the very last one. Performing a couple of Reidemeister moves of type II as shown below, Fig. 5, we have;

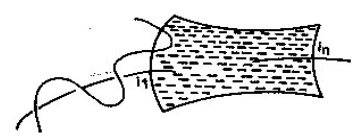

Fig. 5. Performance of reidemeister move of type II.

Now, the crossing $i_{1}$ is a candidate for a Reidemeister move of type III. We perform it and continue performing a finite sequence of suitable Reidemeister moves over all the crossings falling between the crossing $i_{1}$ and $i_{n}$, resulting as; Fig. 6.

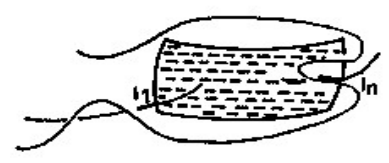

Fig. 6. Performance of a sequence of reidmeister moves.

Now, the crossing $i_{n}$ is a candidate for Reidemeister move of type III; performing this, we have;

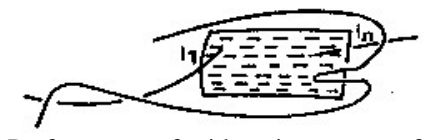

Fig. 7. Performance of reidemeister move of type III.

Now, perform a couple of Reidemeister moves of type II; one can easily achieve as required.

Now, let us observe the effects of $\mathrm{R}$-move on the corresponding graphs of the knot in Fig. 8.

Observe that $\mathrm{R}^{*}$-move changes the black regions into white regions and vice versa. The LR-Graph corresponding to black regions systematically changed to LR-Graph that corresponds to the white regions of the same knot. One can change the dual graphs into one another by the graphic moves [12] resulting from Reidemeister moves. The graph corresponding to black regions systematically changed to the graph that corresponds to the white regions of the same knot producing the equivalent dual graphs. If we consider the labeled graph then the labeling (crossings) also changes 
systematically and accordingly. This gives the following;

Theorem 2. Dual graphs are equivalent via Reidemeister move.

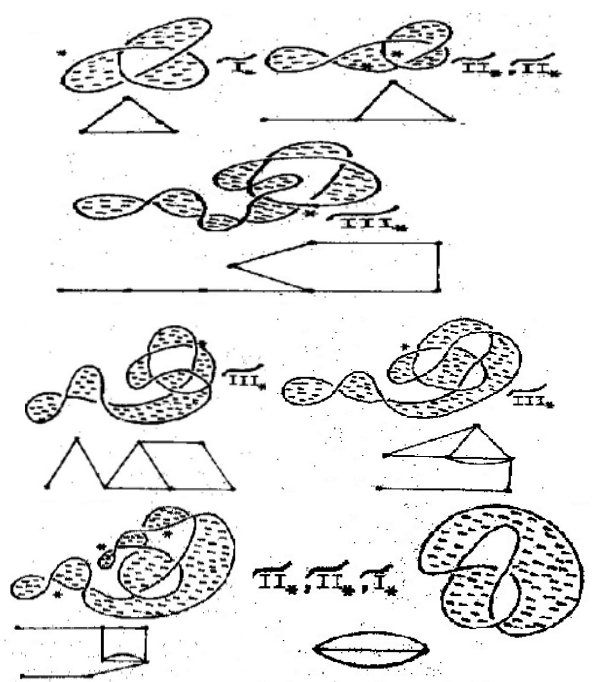

Fig. 8. Effects of $\mathrm{R}^{*}$-move on the corresponding graphs of knot.

\section{REFERENCES}

[1] C. Liang and Y. Jiang, "The chirality of ground DNA knots and links," Journal of Theoretical Biology, vol. 158, no. 2, pp. 231-243, 1992

[2] W. Qiu and H. Xin, "Topological structure of closed circular DNA," Journal of Molecular Structure: Theochem, vol. 428, no. 1-3, pp. 35-39, 1998.

[3] J. Hoste, M. Thistlethwaite, and J. Weeks, "The first 1701936 knots," Math. Intell., vol. 20, pp. 33-48, Fall 1998.

[4] K. Reidemeister, Knotentheorie, Ergebnisse der Mathematik und Ihrer Grenzgebiete, (Alte Folge), Band 1, Heft 1, Springer, Berlin, 1932.

[5] G. Burde and H. Zieschang, Knots, De-Gruyter, 1985.
[6] R. J. Aumann, "Asphericity of alternating knots," Ann. of Math., vol. 64, pp. 374-392, 1956.

[7] R. H. Crowell, "Genus of alternating link type," Ann. of Math., vol. 3, pp. 101-120, 1959

[8] S. Kinoshita and H. Terasaka, "On union of knots," Osaka Math. J., vol. 9, pp. 131-153, 1957.

[9] L. H. Kauffman, "New invariants in the theory of knots," Amer. Math. Monthly, vol. 95, pp. 195-242, 1988.

[10] M. Azram, "Achirality of knots," Acta Mathematica Hungarica, vol. 101, no. 3, pp. 217-226, 2003.

[11] M. Azram, "Achirality via Graphs," Far East Journal of Mathematical Sciences, vol. 38, no. 1, vol. 49-55, 2010.

[12] M. Azram, "An algorithm that changes the companion graphs," Arkive for Matematik, vol. 32, no. 2, pp. 277-291, 1994.

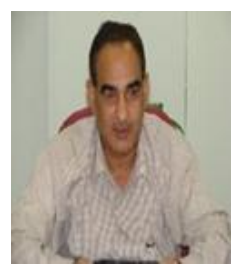

Mohammad Azram received his B.S. and M.Sc. (with distinction) in mathematics from the University of Peshawar, Pakistan, in 1974 and 1976 respectively. $\mathrm{He}$ started his teaching career as a lecturer in the Department of Mathematics, University of Peshawar in 1977. He received his MS and Ph.D in Mathematics from the University of Idaho (USA) in 1985 and 1989 respectively. His Ph.D. thesis (Graph Theoretic Version of Reidemeister Moves) was supervised by the then a leading mathematician Prof. Charles $\mathrm{O}$, Christenson. His research interest is in the area of Low-dimensional Cell-complexes/Knot Theory, Point-set Topology, Mathematical Physics and Topological Algebras. As a teacher, he served the University of Peshawar in the capacity of Lecturer, Assistant Prof, Assoc Prof \& Prof. As a leader/administrator, he served the University of Peshawar as Director (Ph.D./M.Phil programs), Head (Dept of Computer Science) and member (Academic Council, the Senate, affiliation committee \& Peshawar University Teacher's Association). He has joined the International Islamic University of Malaysia in 1999. Apart from many other academic and administrative responsibilities, he has served IIUM as a Professor of Mathematics, Deputy Dean (Academics, Centre for Postgraduate Studies) and Head (Dept of Sc, Faculty of Engg). During his stay at IIUM, he won Two Gold and Two Bronze Medals in research exhibitions. He has also been awarded the best teacher award for the session 2008/09. 